\title{
Novel opportunities and challenges offered by nanobiomaterials in tissue engineering
}

\author{
Fabrizio Gelain \\ Bioscience and Biotechnology \\ Department, University \\ of Milan-Bicocca, Milan, Italy
}

Correspondence: Fabrizio Gelain Bioscience and Biotechnology Department, University of Milan-Bicocca, Piazza della Scienza 2, Milan, Italy Tel +390264483312

$\mathrm{Fax}+390264483314$

Email fabrizio.gelain@unimib.it

\begin{abstract}
Over the last decades, tissue engineering has demonstrated an unquestionable potential to regenerate damaged tissues and organs. Some tissue-engineered solutions recently entered the clinics (eg, artificial bladder, corneal epithelium, engineered skin), but most of the pathologies of interest are still far from being solved. The advent of stem cells opened the door to large-scale production of "raw living matter" for cell replacement and boosted the overall sector in the last decade. Still reliable synthetic scaffolds fairly resembling the nanostructure of extracellular matrices, showing mechanical properties comparable to those of the tissues to be regenerated and capable of being modularly functionalized with biological active motifs, became feasible only in the last years thanks to newly introduced nanotechnology techniques of material design, synthesis, and characterization. Nanostructured synthetic matrices look to be the next generation scaffolds, opening new powerful pathways for tissue regeneration and introducing new challenges at the same time. We here present a detailed overview of the advantages, applications, and limitations of nanostructured matrices with a focus on both electrospun and self-assembling scaffolds.
\end{abstract}

Keywords: self-assembling peptide, electrospinning, tissue engineering, functionalization

\section{Introduction}

Nearly all tissue cells are located in 3-dimensional (3D) microenvironments in the bodies. Cells are surrounded by other cells and/or by the extracellular matrix (ECM): in these microenvironments many extracellular ligands (ie, many types of collagens, fibronectin, laminin, and other matrix proteins) not only allow attachments between cells and basal membranes but also allow access to oxygen, hormones, and nutrients. 3D cell microenvironments comprise a complex network of ECM nanofibers with specific bioactive motifs and nanopores. As a consequence, one of the most important targets in regenerative therapies is to mimic the local microenvironment of tissues by using nanostructured matrices capable of providing 3D supports for stem cells transplanted to injured tissues where cavities or scars thwart any cell therapy based approach. Nanomatrices may also provide more reliable in in vitro models of hybrid tissues, thus allowing fast systematic in vitro screenings if compared to expensive and life-costing animal experiments. Additionally, in the ambitious attempt of regenerating complex tissues, miniaturized scaffold structures are going to give a real chance of developing treatments comprising multiple approaches like controlled drug delivery, implants mechanically responsive to external stimuli, stem cell technology, in situ gene therapy and so on. The field is undergoing a rapid growth and it is impossible to cover it comprehensively in a few pages, thus, in this review, we mainly focus on researches related to the synthesis and the application of nanostructured scaffolds in cell therapies, a rapidly growing area of nanomedicine and one of the fields of expertise of our lab. Readers interested in advances in nanobiomaterials as anticancer agents or generic drug delivery carriers like nanoparticles or nanovesicles can consult other 
helpful reviews published in previous issues of this journal (Basarkar and Singh 2007; Douziech-Eyrolles et al 2007; Haddish-Berhane et al 2007).

\section{The "sense of touch" of cells}

It has getting accepted among the scientific community that flat glasses or plastic substrates employed in in vitro experiments are not representative of tissue microenvironments located in organisms. Indeed, tissue-specific architectures, biomechanical forces, cell-cell interactions, and cytokine diffusion gradients are poorly reproduced in 2-dimensional (2D) surfaces. Cells cultured in monolayers often modify their intrinsic signal pathways, thus endothelial cells in 3D experimental in vitro models may provide biased outcomes far from those found in in vivo experiments (Gomez-Lechon et al 1998). In more detail, cells perceive chemical and physical cues. Chemical cues, for the most part, comprise biomolecules available for binding to cell membrane receptors or hydrophilicity modifications of the substrates involved (Jansen et al 2005). Physical cues, on the other hand, can be micro- and nanolevel modifications of the scaffold structures. The most widely known example of response to these physical cues is the cells' ability to recognize topographical differences, such as presence of grooves and ridges, and their tendency to be guided by these features (Walboomers and Jansen 2001). Indeed cell adhesion to a carrier is a crucial step towards the development of an effective regenerative approach. Cellular responsiveness to nanoscale topography was demonstrated with several cell types, such as corneal epithelial cells (Dalby et al 2004), meningeal cells (Manwaring et al 2004), and fibroblasts (Gallagher et al 2002). More interestingly, diverse cell types reacted to the same topography in markedly different ways (Gallant et al 2007). Other groups showed how topographical features like nanopillars or pits prevented cell adhesion (Dalby et al 2004; Wan et al 2005). An interesting application that may arise from these findings is for vascular stents, where the ability to deter cells from adhering to the implant surface is an advantage. Since nanoscale features are much smaller than the cell dimensions, they are likely to trigger the formation of focal adhesions or even single binding of membrane proteins. Thus, nanoscale patterns might allow an unprecedented precise control of cell directionality and migration in implants and ex vivo devices. A set of investigations compared the proliferation of cells attached to nanofibers with that one of cells attached to smooth films composed of the same biomaterial. Cell adhesion and proliferation were improved with nanofibrous scaffolds in the majority of cases (Xu et al 2004).
In the last few years, new breakthroughs have pointed out the importance of conducting cell experiments in 3D scaffolds made of nanofibers capable of evenly wrapping cell bodies and branches. In a milestone work, Discher and colleagues (Engler et al 2006) demonstrated how the lineage of mesenchimal stem cells can be influenced by the mechanical properties of a synthetic matrix. By varying the matrix physical stiffness mesenchimal stem cells were selectively differentiated into osteoblasts, neurons, and myoblasts. Moreover, the mechanical stiffness of their nanostructured scaffolds was shown to influence capillary morphogenesis of human umbilical endothelial cells in 3D vitro experiments (Sieminski et al 2007), giving evidence of how more malleable substrates are preferred for capillary morphogenesis. In a work of Ghosh and colleagues (2005) the authors showed how melanoma cells have different gene expression profiles depending on culture conditions: from monolayer cell cultures on $2 \mathrm{D}$ surfaces to spheroids in $3 \mathrm{D}$ scaffolds. Nicely they showed that some genes upregulated in tumor biopsies are upregulated in 3D melanoma cell cultures as well, but not in 2D cultures. These works are likely to open the door to new basic science understandings offered by 3D cell culture paradigms and, as a consequence, by nanostructured biomaterials.

Notwithstanding the potential of nanostructured scaffolds, two additional issues have to be considered to obtain feasible tissue engineering approaches: 1) scaffold functionalization with bioactive motifs and 2) projecting and synthesizing matrices with well-defined 3D nanoand microstructures. Biochemical stimuli are also crucial to foster cell adhesion and stem cell differentiation. The option of providing scaffolds with various biochemical cues capable of interacting with cell membrane receptors is the underlying potential of the overall peptidomimetic literature. Indeed, natural polymers, such as collagens, fibronectin, and hyaluronic acid are usually superior over traditional synthetic polymers used in tissue engineering in stimulating cell adhesion since they inherently possess specific amino-acid sequences that can be recognized by the cells (Gelain et al 2007).

Conventional scaffold manufacturing techniques, such as solvent casting, salt leaching, and freeze drying, produce scaffolds in which individual nanostructures are randomly oriented. Thus, even though carriers may induce adhesion and/or proliferation of cells, the obtained cytoarchitecture would remain random on these scaffolds. However in many tissues the 3D spatial cell organization is crucial for the proper functionality of the tissues themselves: ie, in the 
case of central nervous tissue an uncontrolled sprouting of regenerated fibers may be life-threatening for a patient with spinal cord injuries. In the natural corneal stroma, cells inhabit an environment that is composed of aligned fibrils of collagen: this specific orientation is shown to be essential for the transparency of the cornea and an artificial cornea has likely to show similar optical properties. In this regard, nanostructures suitable for multiple biological functionalizations, with tailorable mechanical properties and well defined 3D structure orientation, are nanofibrous scaffolds.

\section{Nanofiber scaffolds}

The word nanofiber generally refers to a fiber having a diameter less than $100 \mathrm{~nm}$, but by definition, fibers with diameters less than $1000 \mathrm{~nm}$, produced via some ultrafine manufacturing techniques such as electrospinning, are also classified as nanofibers. Among the main advantages of nanofiber scaffolds it is important to remind their high surface area to volume ratio, their suitability for high-density functionalization, their high diffusive capacities and unconventional mechanical properties. Industrial nanotechnology research developed various fabrication methods for obtaining nanofibers: drawing, phase separation, melt-blowing, template synthesis, electrospinning, and self-assembly. However, only electrospinning and self-assembly achieved a widespread usage for regenerative medicine approaches thanks to their versatility and fascinating potential (Zhang et al 2005; Guo et al 2007; Panseri et al 2008; Tysseling-Mattiace et al 2008). Research efforts deploying these two nanotechnology techniques are indeed focused on investigating their potential for tissue engineering of cartilage, bone, nerve, skeletal muscle, and skin and blood vessels (Yoshimoto et al 2003; Zong et al 2005; Riboldi et al 2008).

\section{Electrospun scaffolds}

In electrospinning, a strong electrical field is applied to a droplet formed from a polymer solution. The charging of the fluid leads to a deformation of the droplet and to the ejection of the fluid jet from the tip of the cone. The charged jet is accelerated toward the counter electrode, and it thins rapidly during this period due to droplet elongation and evaporation of the solvent until solid or wet fibers are deposited onto a substrate, usually a planar surface, located on top of the counter electrode. Electrospinning process is economical, simple, yields continuous fibers (while for self-assembling they are a few micrometers in length) and hollow fibers, and is versatile enough to be applied to a variety of materials to obtain knitted structures (Bini et al 2004), aligned nanofibers
(Corey et al 2007), or core-shell structures. In electrospinning, fibers can be spun from polymers at their molten state or organic solvents and water polymeric solutions. Nanofibers with nanopores can also be produced by choosing single solvents causing phase separations into solvent-rich (ie, pore forming) and solvent-poor areas within the jet or ternary solutions containing two different polymers that phase separate within the fibers. This technique is suited for incorporating chromophores, drugs, enzymes, or semiconductor and magnetic nanoparticles directly during the spinning process.

Noteworthy core-shell fibers produced via coelectrospinning are particularly interesting for drug-delivery scaffolds: cores control the storage of the functional materials to be released and shells the release kinetics. Two dies are arranged in this case in a coaxial double injector and both holes are connected to two reservoirs containing different spinning solutions (Dror et al 2007). Cores can be a fluid to provide a natural environment for the biological object while the electric charges are located practically only at the outer shell surface, so that the inner droplet is not charged at all. Other studies demonstrated that biological subjects such as proteins, viruses, or bacteria will not experience electrical stresses and that the mechanical stresses can be adjusted to stay moderate during co-electrospinning.

Several approaches have been developed to enhance the mechanical properties of electrospun scaffolds: induction of a chosen 3D orientation of nanofibers or even knitting whole meshes, nanofibers annealing at high temperatures (to reduce crystal defects and to increase crystal sizes); fiber cross-linking by subsequent irradiation or chemical reactions (Bini et al 2004; Xu et al 2007).

In vitro cell culture experiments have been carried out with fibers of biocompatible and biodegradable polymers of natural and synthetic origin such as poly(glycolic acid) (PGA), poly(1-lactic acid) (PLLA), poly( $\varepsilon$-caprolactone) (PCL), as well as copolymers from the corresponding monomers in various compositions, segmented polyurethanes, polyphosphazenes, gelatin, collagen, and chitosan (Chen et al 2008; Choi et al 2008; Corey et al 2008; Powell and Boyce 2008). A broad spectrum of cells has been seeded on electrospun scaffolds: for instance keratinocytes, chondrocytes, osteoblasts, mesenchymal stem cells, Schwann cells, neural stem cells, smooth muscle cells, osteoblasts, fibroblasts, and endothelial cells. Target organs or tissues to be regenerated via electrospun scaffolds included bone, skin, neural tissue, cartilage, as well as blood vessel, heart, or lung (Pham et al 2006; Rubenstein et al 2007; Chew et al 2008; Venugopal et al 2008). In particular, electrospun nano/microstructured 
PLGA-based scaffolds have been shown to provide both guidance and flexibility to cardiac myocytes (Zong et al 2005). Co-cultures of keratinocytes, skin fibroblasts and endothelial cells revealed a synergistic effect given by the different co-cultured cell phenotypes: cell proliferation and self-organization of tissue constructs were enhanced and a native epidermal-like structure formation took place (Sun et al 2005).

In the last decades most of the strategies adopted in nervous tissue engineering made use of synthetic or biologically derived channel guides that eventually cause cell loss due to unphysiological local stresses exerted over nervous tissue during patient's movement. In electrospinning cylindrically shaped rotating electrodes can be employed to collect the fibers, either parallel or random depending on the rotation speed or other electric field parameters, to obtain fibrous tubular arrangements. Electrospinning technique makes it possible to spin micro-braided microfiber and nanofiber flexible tubular scaffolds composed of a number of natural and synthetic components, showing high porosity, remarkable surface/volume ratio, and satisfactory mechanical properties (Bini et al 2004, 2005). In our group (Panseri et al 2008), we studied the joint advantages achievable by using multiscaled tubular structures made of electrospun microfiber and nanofiber as nerve tubular guides (see Figure 1). A detailed analysis of various aspects of nerve regeneration showed how microfibrous and nanofibrous prostheses did not produce mechanical stress-related nervous degenerations and, on the other hand, favored functional and effective nervous regeneration by providing a nanostructured inner cavity for the regenerating fibers and maintaining the nervous chamber isolated from the surrounding tissues. Further enhancements to the proposed scaffold can be adopted in the near future: instead of adopting randomly oriented fibers, nanofibers aligned along the longitudinal axis of the nerve guides can be preferred to optimize the amount of polymer necessary to preserve the nerve chamber mechanical properties.

Though many potential applications of electrospinning are being investigated, it is worth mentioning some drawbacks: the harshness of the overall process and the presence of harmful organic chemical solvents preclude any possible addition of cells while the scaffold is forming. Attempts were made to seed scaffolds in dynamic conditions or just onto their surfaces (layer by layer seeding), but, even if a considerable cell migration occurred days and weeks after seeding, uniform cell distributions throughout the 3D scaffolds are rather difficult to obtain (Srouji et al 2008). Indeed cell ingrowth is still usually restricted to a depth of a few $100 \mu \mathrm{m}$ in nonwoven meshes and scaffold porosity continue to be problematic for cell migration in such nanofiber-based scaffolds. The exact control over average pore diameters and distribution in a 3D matrix is still a subject for further optimizations. Nonetheless, a technique that effectively solved the problem of achieving an even 3D scaffold cell distribution is self-assembling.

\section{Self-assembling peptide scaffolds}

Self-assembling peptides (Sapeptides) have been studied for decades but only in the last 15 years they were classified as biomaterials and substrates for regenerative medicine. Sapeptides

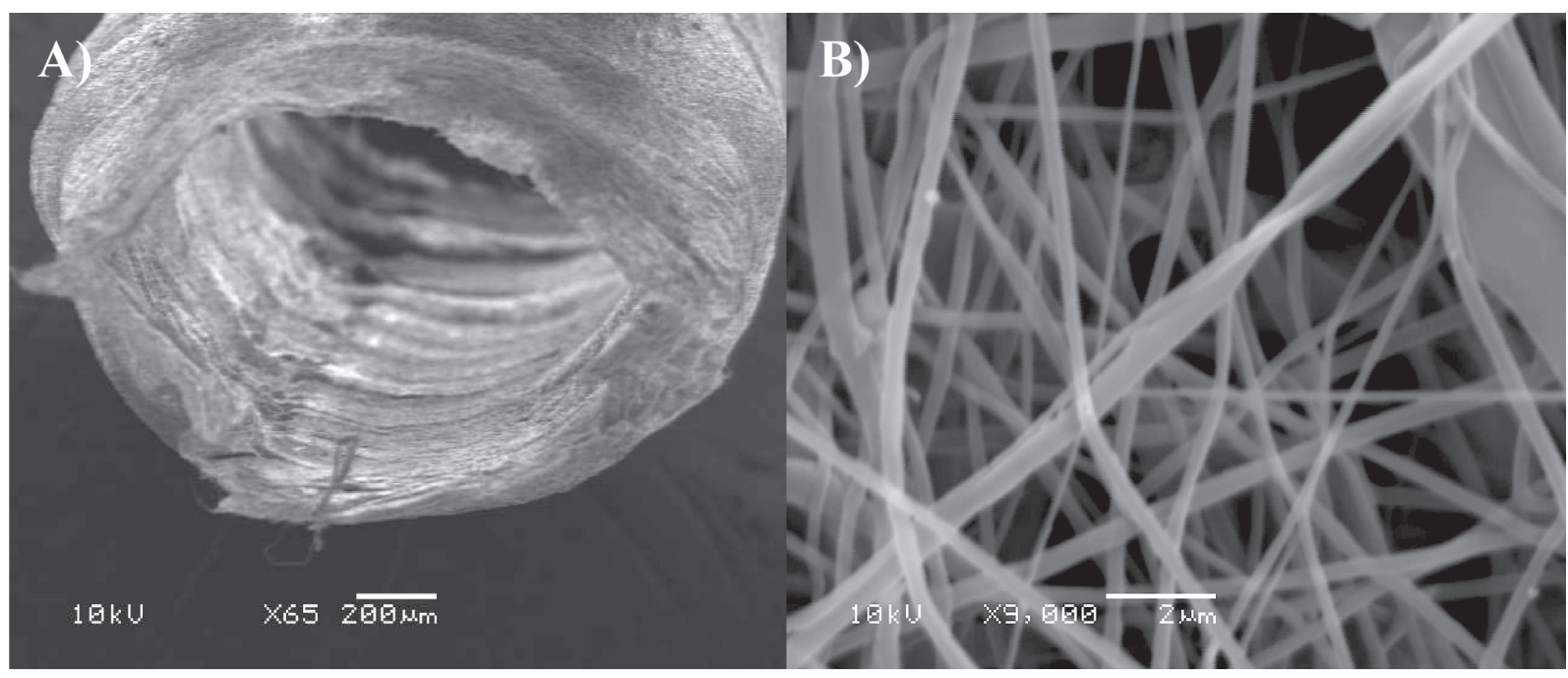

Figure I SEM imaging of micro- and nanofiber electrospun PCL/PGLA tubular scaffolds designed for regenerating sciatic nerve transections. A) Tube lumen and B) zoomed details of the tube wall. Both nano- and microfibers are visible. Fiber links are obtained via partial solvent evaporation and polymer annealing subsequent to electrospinning in order to increase the overall prosthesis mechanical properties (image by courtesy of Joseph Lowery). 
generally show hydrophilic heads with hydrophobic tails or, alternatively, periodic repeats of alternating ionic hydrophilic and hydrophobic amino acids, with both molecular types spontaneously forming $\beta$-sheet structures. They are isobuoyant in aqueous solution and readily transportable to different environments. Upon exposure to neutral $\mathrm{pH}$ aqueous solutions, ions screen the charged peptide residues and hydrophobic residues (constituting the nonpolar surfaces of $\beta$-sheets) of different $\beta$-sheets can pack together thanks to their hydrophobic interactions in water, thus giving doublelayered $\beta$-sheet nanofibers (Zhang 2003), structures that are found in silk fibroin from silkworms and spiders, or $\beta$-sheets assemblies parallel to cylindrical nanofibers (Silva et al 2004). Remarkably, sapeptide scaffolds, with great than $99 \%$ water content, show nanostructures resembling those of ECM derived substrates like Matrigel ${ }^{\mathrm{TM}}$ (BD Biosciences, San Jose, CA, USA) (Gelain et al 2006). Since the building blocks of self-assembling peptide scaffolds are natural amino acids and their degradation products can be reused by the body, unlike most of the other synthetic biomaterials, they have been shown to elicit a negligible immune response and poor inflammatory reaction in in vivo experiments (Davis et al 2005, 2006; Ellis-Behnke et al 2006). The self-assembling step is therefore harmless and the versatility of sapeptide structures is comparable to that one of proteins.

Thanks to their remarkable biocompatibility and their potential for easy and modular design, sapeptides are suited for 3D cell biology studies, controlled drug delivery, and tissue engineering applications. However the theory underlying the self-assembling process still have to provide comprehensive designing rules capable of fitting together various needs coming from the heterogeneous plethora of possible applications.

\section{Self-assembled peptide matrices for in vitro applications}

Three-dimensional self-assembling scaffolds have become increasingly important in in vitro experiments as reliable models to predict cellular response to drugs or physical stimuli. For instance sapeptide scaffolds were shown to stimulate neurite outgrowth (Holmes et al 2000), neural stem cell differentiation (Gelain et al 2006), and have been adopted in cardiomyocytes, osteoblasts (Horii et al 2007), and chondrocytes cultures (Kisiday et al 2002).

KLD12, belonging to the class of double layer $\beta$-sheets forming peptides, was used to encapsulate chondrocytes within peptide scaffolds for cartilage tissue engineering (Kisiday et al 2002). Kisiday and colleagues (2002) also reported that Grodznisky and collegues, after culturing chondrocytes embedded in KLD12 scaffolds, detected a cartilage-like ECM indicative of a stable chondrocyte phenotype matrix synthesis. Time dependent accumulation of proteoglycans-rich ECM was paralleled by increases in material stiffness indicative of deposition of mechanically functional tissue. This seminal work confirmed the promising opportunity for cartilage repair given by self-assembling peptide 3D matrices as scaffolds for the synthesis and accumulation of functional ECM to regenerate connective tissues.

In a work of Holmes and colleagues (2000), RADA16-I and RADA16-II $\beta$-sheets forming peptides formed nanostructured matrices capable of stimulating extensive rat neurite outgrowth and active synapses formation. The high impact of their findings is based on the following rationale. Usually neuronal primary cultures rely upon usage of biological derived substrates to obtain in vitro long-term cultures of neuronal networks with the highest survival and neural network activity. This is crucial to achieve a number of goals: 1) testing new brain-machine interfaces for chronic stimulation; 2) a reliable in vitro model for release tests of neurological drugs; 3 ) the creation of hybrid devices comprising synthetic and/or biological-based computing components; 4) a better understanding of nervous tissue signal-processing mechanisms (Mann-Metzerand Yarom 2002); and so on. Unfortunately, in long-term cell cultures, cell detachment is usually mediated by substrate de-adsorption and different substrate batch preparations may impair the reproducibility of experimental results. A synthetic 99.5\% pure substrate suitable to be linked to a bottom well surface via self-assembling or chemical reactions and functionalized with bioactive motifs may be the gold standard for neuronal cultures and differentiated progeny tests of human neural stem cells.

By systematically comparing neural stem cell (NSC) adhesion and differentiation over various substrates (Gelain et al 2007), we confirmed that natural derived substrates showed the best performances and RADA16-I sapeptide coaxed neural stem cell differentiation and survival similarly to other synthetic biomaterials commonly used in tissue engineering. Although sapeptides are promising scaffolds, they usually show no specific cell-biomaterial interaction because their self-assembling sequences are not found in living systems (RAD mimic RGD but is far different from the ubiquitous integrin receptor-binding site). Nonetheless the hypothesis of scaffold functionalization with short bioactive motifs is extremely feasible with sapeptides. As bioactive motifs consist of short peptide sequences they can 
be synthesized and linked to the self-assembling "cores" as part of the peptides themselves without any post-synthesis additional chemical reaction. A significant enhancement of cells and tissues interactions with self-assembled scaffolds was obtained by adding short peptide sequences chosen from a wealth of peptide functional motifs studied in the peptidomimetic literature (Ayad et al 1998; Kreisand Vale 1999; Ricard-Blum et al 2000). Consequently, some groups focused their attention towards sapeptides functionalized with laminin-derived motifs (Silva et al 2004) or evaluated fibronectin and collagens derived functional motifs extended from RADA16-I-based peptides (Genove et al 2005).

Our group functionalized the RADA16-I sapeptide at the C-termini (Gelain et al 2006): a spacer (eg, two glycines residues) was added to provide sufficient exposure of the added motifs to cell membrane receptors. Upon scaffold self-assembling the added motifs flagged from the newly formed nanofibers. To note, double functionalization (on either sides of the peptide) or N-termini functionalization are alternative or complementary strategies. Nanofibers took part to the overall scaffold architecture thus giving microenvironments functionalized with specific biological stimuli. In such a designed self-assembled 3D microenvironment, the functional motifs were linked harmlessly to the scaffold nanofibers embedding cell bodies in all dimensions.

Different functional motifs can be incorporated in various ratios in the same scaffold if the self-assembling "core" is maintained regardless of the functionalization itself. We functionalized self-assembling scaffolds with bone marrow homing peptides (Gelain et al 2006), functional motifs discovered to be particularly promising for stimulating NSC adhesion and differentiation (see Figure 2). Biomaterial functionalization is usually achieved for flat or grooved surfaces and microstructured scaffolds: just recently PLGA or PCL nanofibers became commercially available for functionalization with a limited number of biological motifs via a biochemical process involving potentially harmful chemicals and, most importantly, far from being adopted to tightly embed living cells in a functionalized 3D matrix. Indeed self-assembling scaffolds show the unquestionable synergic advantages of both spontaneously forming scaffolds embedding cells, thus placing functional motifs close to cell membrane receptors, and selectively stimulating diverse cell signaling pathways by choosing various sets of functional motifs to be included in the 3D scaffold nanostructures (see Figure 3). In 3D scaffolds, cells receive more dense and evenly distributed amount of functional motifs available for cell membrane receptor binding than when in contact with coated 2D RGD-coated surfaces

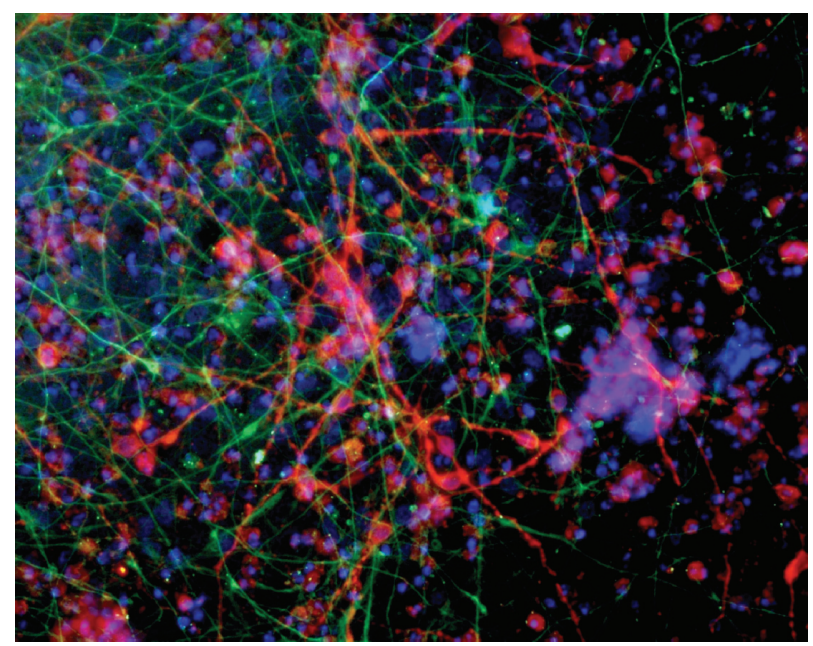

Figure 2 Human Neural Stem Cells cultured in a RADAI6-I-BMHPI 3D scaffold ( 3 weeks in vitro). Cell nuclei are stained with DAPI (blue), neurons with $\beta$ Tubulin antibody (red), and astrocytes with GFAP antibody (green). In this long-term cultures neuronal morphologies resemble fairly mature neurons. A highly connected neuronal network is shown. Branched astrocytes also give evidence of differentiation of part of the stem cell progeny toward the astroglial phenotype.

or microfibers. A specific set of different motifs can be easily tested for selective gene expression activation: for example, we evidenced how neural stem cells differentiation in BMHP1 functionalized scaffolds highly increased the mRNA expression of Laminin-b2 and Fibulin 1 genes, both proteins involved in ECM assembly, suggesting the existence of a possible common pathway activation. Similar selective stimulations cannot be obtained, and neither tuned, with any biologically derived substrates used for in vitro neural cultures. This and other studies may uncover new insights into cell expression mechanisms powerful for basic science purposes.

Horii and colleagues (2007) designed other selfassembling scaffolds functionalized for osteoblast cultures. Functional motifs included osteogenic growth peptides, osteopontin cell adhesion motifs, and 2-unit RGD binding sequences. Compared to pure RADA16-I scaffolds, functionalized self-assembled matrices significantly promoted mouse pre-osteoblast cell proliferation, osteogenic differentiation (early and late secretion product markers for osteoblastic differentiation were significantly increased) and cell migration into the self-assembled 3D scaffolds. These results suggest that designer peptide scaffolds may be useful for bone tissue regenerative applications as well.

\section{In vivo applications of self-assembling peptides}

More and more groups have been testing the innovative potential of sapeptides for regenerative medicine as reliable, 


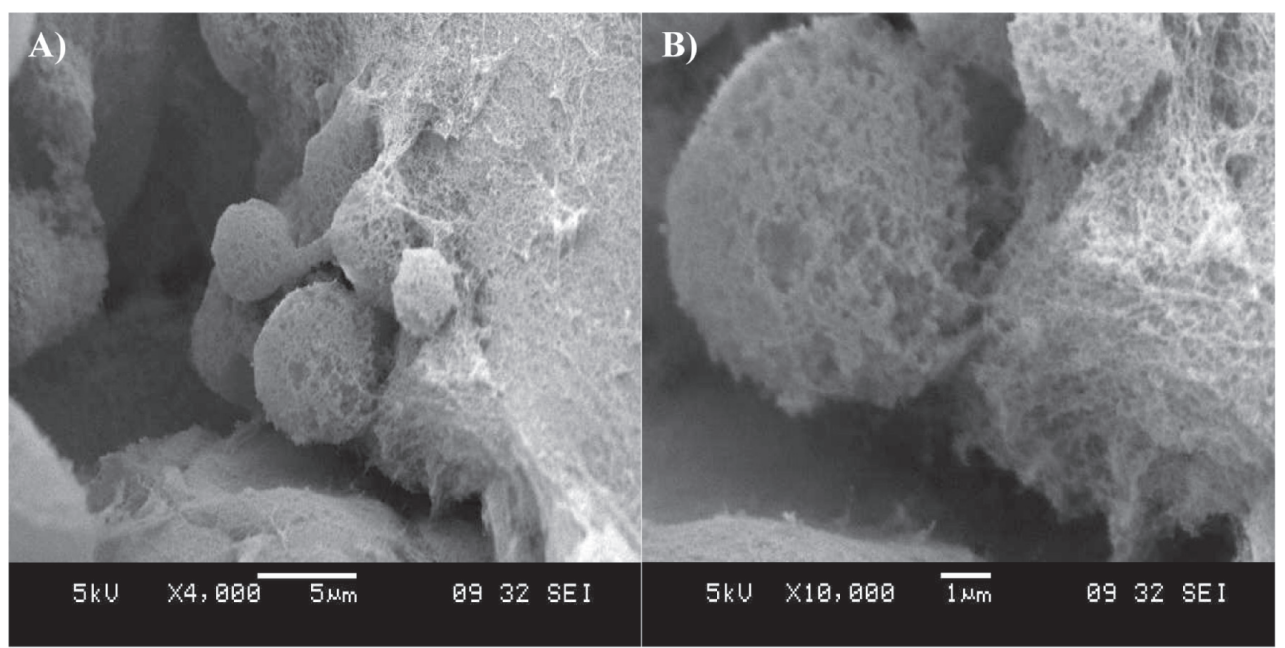

Figure 3 SEM imaging of a cluster of neural stem cells cultured in a RADA I6-I-BMHPI self-assembled scaffold. Low- (A) and high-magnification (B) images highlight cell bodies partially but tightly wrapped with functionalized nanofibers.

safe and easy-to-tailor scaffolds. In particular, Ellis-Behnke and colleagues (2006) showed how injections of RADA16-I sapeptide enhanced neural regeneration in optic nerve surgical lesions in hamster pups while in sham-operated animals the functional damage was still evident after weeks. Additionally, by using neural tracing techniques, Ellis-Behnke and colleauges (2006) demonstrated how retinal axon projections regenerated through the injury site, reconnecting the rostral nervous tissue to the superior colliculus caudal to the lesion. In all treated animals, a significant sight recovery was proved via a specific behavioral test. The mechanism responsible for this remarkable outcome still has to be clearly elucidated. The authors argue that nervous regeneration may rely upon the presence of specific chemical cues like active motifs and chemotactic agents that are spontaneously released by host tissues surrounding the site of injury during the follow up subsequent the acute nervous trauma (Busch and Silver 2007) and trapped within the self-assembled scaffolds. Indeed peptide nanofibers can spontaneously adsorb and then slowly release various biomolecules (Nagai et al 2006), thus providing an enduring chemotactic effect. Furthermore, the gelation process, taking place within the injured cavity, may be beneficial to the establishment of tight and continuous scaffold-tissue junctions helpful in avoiding the formation of cavities normally found in the case of preformed scaffold implants. In nervous system a crucial role during the damage process is usually given by the secondary damage following the initial injury: this comprises a complex multi-step inflammatory cascade that can even two-fold increase the initial damage entity. Interestingly, Ellis-Behnke and colleagues (2006) showed an inflammatory reaction to their implants lower than in sham-operated animals, thus letting the authors infer a decreased host tissue response, to the initial injury, given by the implanted self-assembled scaffolds. The nanostructured matrices may act as physic and chemical barriers for the inflammatory cells preventing their gathering at the injury site and, as a consequence, their subsequent chemotactic action for other cells involved in the inflammatory reaction. Similar hypotheses of the self-assembling nervous regenerative potential were formulated by Ellis-Behnke and Stupp in their most recent works in spinal cord regeneration (Guo et al 2007; Tysseling-Mattiace et al 2008).

Sapeptide scaffolds were used to regenerate cardiac tissue: Lee's group injected embryonic stem cells suspended in RADA16-II peptide solutions into the myocardium of mice (Davis et al 2005). They demonstrated that sapeptides can be easily injected into myocardia to create $3 \mathrm{D}$ microenvironments. Self-assembled scaffolds recruited both endogenous endothelial and smooth muscle cells while exogenously injected cells survived in the recreated microenvironments. Additionally, self-assembled microenvironments induced vascularization, a crucial step towards any effective regenerative therapy. Thereafter, Lee's group biotinylated RADA16-II peptide to link IGF-1 to the selfassembled scaffolds prior injection into the myocardium. Thanks to their biotin sandwich strategy they achieved an in situ slow release of IGF-1 in infarctuated myocardia of rats (Davis et al 2006). By adding cardiomyocytes to the IGF-1 loaded scaffolds they showed how cell therapy combined with IGF-1 delivery via biotinylated nanofibers significantly improved systolic function in infarctuated myocardia. 
Beyond tissue engineering applications, Ellis-Behnke and colleagues (2006) serendipitously discovered that self-assembling peptides establish nanofiber barriers capable of achieving complete hemostasis. They applied the self-assembling peptide solution directly to wounds located either in the brain or spinal cord, liver, femoral artery, and skin of mammals. Depending on the wound tissue location to be healed, and consequently on the pressure of the blood flow at the injury site, different amounts of sapeptide solution were injected at different concentrations to evenly cover the wounded area and to provide an initial mechanical strength of the peptide matrix sufficient to stop the blood flow while the gelation of the overall scaffold occurred. Noteworthy, while clinically used agents usually take hundreds of seconds to achieve homeostasis, bleeding was stopped almost instantly with scaffold placement and the effect endured even after scaffold removal. Moreover, a practical advantage is given by the intrinsic transparency of the hemostatic scaffolds (a quality not shared with any other hemostatic agents), allowing surgeons to easily monitor the bleeding at the injury sites. RADA16-I stopped bleeding without the use of coagulation, cauterization, vasoconstriction, pressure, or cross-linked adhesives. Even if the exact mechanism underlying hemostatic property of RADA16-I solutions was still not fully understood the authors suggested that sapeptide solution form a barrier, stemming the blood flow and facilitating the migration of cells neighboring the injury site to repair the wound itself. Being well accepted among the scientific community that tight contacts between wounds and hemostatic agents are crucial to achieve hemostasis, the authors, via transmission electron microscopy imaging, proved the existence of tight contacts between tissue-specific cells, red blood cells, and the injected scaffolds. They showed close interfaces resembling those found between extracellular matrices and cells located in healthy tissues. Additionally it has to be mentioned that stiffer self-assembled scaffolds were not necessarily more effective hemostatic solutions: indeed some of the self-assembling scaffolds they tested proved the opposite. The compliance of the whole scaffold with the tissue to be healed is crucial to preserve thigh cellscaffold contacts with the injured tissues: because of blood flow pulses in arteries or local movements of the overall organs a too stiff and fragile scaffold indeed goes into pieces leaving the blood flow free to bleed shortly after gelation. The mechanical stiffness of the self-assembled scaffolds is strictly dependent on the peptide sequences themselves (Caplan et al 2002); this is just an example of how improved knowledge over self-assembling theory will be essential to develop a first aid medical device available and cheap for everybody in the future by 1) optimizing a specific sapeptide sequence capable of achieving hemostasis almost instantly and with the minimum solution amount, 2) designing an hemostatic solution "ad hoc" for each wounded tissue paying attention to its mechanical properties, blood capillarization, and accessibility via surgeries. Indeed the leading author claimed that the tested sapeptide solutions would likely reduce the quantity of blood needed during surgeries in the future. However, much work has to be done prior their testing in clinical models: as an example we may think of the average higher blood pressure of humans than its values in mice and rats, ie, more demanding conditions are going to be faced in the perspective of their real application in clinics.

Sapeptides may be useful bio-reabsorbable scaffolds for tissue engineering applications in order to alleviate and treat a number of trauma and degenerative diseases, however some drawbacks have to be mentioned in their case too. Currently, unlike electrospun matrices, self-assembled scaffold nanostructures are mainly randomly oriented in the three dimensions: thus monolayers, randomly oriented nonwovens or fractal flat nanostructures have been obtained with selfassembling peptides despite the fact that further efforts have been undertaken to influence the formation of self-assembling structures via electromagnetic fields or microfluidic chambers. This issue may be crucial in tissues where a particular regenerated cytoarchitecture at both nano- and microscale have to be achieved (see previous sections). Some selfassembling peptides requires the addition of salt solutions for self-assembling to take place, this could be particularly harmful with some tissues to be regenerated (Guo et al 2007). Last but most important issue, the majority of the widely used sapeptides spontaneously form $\beta$-sheets structures with poor elastic properties at the mesoscale when compared to those of tissues/organs like skin, lung, and muscles. Indeed, once assembled, sapeptides can go through fragile fragmentations if subjected to important deformations or stresses thus giving fractures and holes in their scaffold microstructure capable of preventing the success of the overall regenerative therapy.

\section{Conclusions}

Nanostructured scaffolds opened the door to new approaches that were difficult to imagine some decades ago. The broad spectrum of promising applications involving electrospun scaffolds and self-assembling peptide matrices testifies their potential for in vitro widespread usage for cell-ECM or cell-cell interaction studies, 3D cell migrations and morphogenesis assays, cancer and stem cells cultures, and, 
more generally, cell response investigations to diverse biochemical and/or physical stimuli. New applications have been discovered and more are likely to come: yet neither of the mentioned nanotechnology scaffold processing techniques is the optimal strategy for a successful regenerative approach near to clinics. Various researchers focus on solving most of the mentioned issues troubling their future applications in regenerative medicine: however it is author's opinion that a joint approach choosing composite nanostructured scaffolds, ie, made of both electrospun and self-assembling nanofibers, may bring together the potential of both strategies giving directionality to tissue regeneration, harmless scaffold functionalization, mechanical compliance, and satisfactory biocompatibility. Nonetheless it is widely accepted that nanostructured scaffolds, a turning point for tissue engineers, will bring regenerative medicine to a new brilliant era

\section{Disclosure}

The author reports no conflicts of interest in this work.

\section{References}

Ayad S, Boot-Handford RP, Humphreise MJ, et al. 1998. The extracellular matrix: Facts Book. San Diego: Academic Press.

Basarkar A, Singh J. 2007. Nanoparticulate systems for polynucleotide delivery. Int J Nanomedicine, 2:353-60.

Bini TB, Gao S, Xu X, et al. 2004. Peripheral nerve regeneration by microbraided poly(L-lactide-co-glycolide) biodegradable polymer fibers. J Biomed Mater Res A, 68:286-95.

Bini TB, Gao S, Wang S, et al. 2005. Development of fibrous biodegradable polymer conduits for guided nerve regeneration. J Mater Sci Mater Med, 16:367-75

Busch SA, Silver J. 2007. The role of extracellular matrix in CNS regeneration. Curr Opin Neurobiol, 17:120-7.

Caplan MR, Schwartzfarb E M, Zhang S, et al. 2002. Effects of systematic variation of amino acid sequence on the mechanical properties of a self-assembling, oligopeptide biomaterial. J Biomater Sci Polym Ed, 13:225-36.

Chen F, Li X, Mo X, et al. 2008. Electrospun chitosan-P(LLA-CL) nanofibers for biomimetic extracellular matrix. J Biomater Sci Polym Ed, 19:677-91.

Chew SY, Mi R, Hoke A, et al. 2008. The effect of the alignment of electrospun fibrous scaffolds on Schwann cell maturation. Biomaterials, 29:653-61.

Choi JS, Lee SJ, Christ GJ, et al. 2008. The influence of electrospun aligned poly(varepsilon-caprolactone)/collagen nanofiber meshes on the formation of self-aligned skeletal muscle myotubes. Biomaterials, 29:2899-906.

Corey JM, Lin DY, Mycek KB, et al. 2007. Aligned electrospun nanofibers specify the direction of dorsal root ganglia neurite growth. $J$ Biomed Mater Res A, 83:636-45.

Corey JM, Gertz CC, Wang BS, et al. 2008. The design of electrospun PLLA nanofiber scaffolds compatible with serum-free growth of primary motor and sensory neurons. Acta Biomater, 4:863-75.

Dalby MJ, Pasqui D, Affrossman S. 2004. Cell response to nano-islands produced by polymer demixing: a brief review. IEE Proc Nanobiotechnol, 151:53-61.
Dalby MJ, Riehle MO, Sutherland DS, et al. 2004. Fibroblast response to a controlled nanoenvironment produced by colloidal lithography. J Biomed Mater Res A, 69:314-22.

Davis ME, Motion JP, Narmoneva DA, et al. 2005. Injectable selfassembling peptide nanofibers create intramyocardial microenvironments for endothelial cells. Circulation, 111:442-50.

Davis ME, Hsieh PC, Takahashi T, et al. 2006. Local myocardial insulin-like growth factor 1 (IGF-1) delivery with biotinylated peptide nanofibers improves cell therapy for myocardial infarction. Proc Natl Acad Sci US A, 103:8155-60.

Douziech-Eyrolles L, Marchais H, Herve K, et al. 2007. Nanovectors for anticancer agents based on superparamagnetic iron oxide nanoparticles. Int J Nanomedicine, 2:541-50.

Dror Y, Salalha W, Avrahami R, et al. 2007. One-step production of polymeric microtubes by co-electrospinning. Small, 3:1064-73.

Ellis-Behnke RG, Liang YX, Tay DK, et al. 2006. Nano hemostat solution: immediate hemostasis at the nanoscale. Nanomedicine, 2:207-15.

Ellis-Behnke RG, Liang YX, You SW, et al. 2006. Nano neuro knitting: peptide nanofiber scaffold for brain repair and axon regeneration with functional return of vision. Proc Natl Acad Sci U S A, 103:5054-9.

Engler AJ, Sen S, Sweeney HL, et al. 2006. Matrix elasticity directs stem cell lineage specification. Cell, 126:677-89.

Gallagher JO, McGhee KF, Wilkinson CD, et al. 2002. Interaction of animal cells with ordered nanotopography. IEEE Trans Nanobioscience, 1:24-8.

Gallant ND, Charest JL, King WP, et al. 2007. Micro- and nano-patterned substrates to manipulate cell adhesion. J Nanosci Nanotechnol, 7:803-7.

Gelain F, Bottai D, Vescovi A, et al. 2006. Designer self-assembling Peptide nanofiber scaffolds for adult mouse neural stem cell 3-dimensional cultures. PLOS ONE, 1:e119.

Gelain F, Lomander A, Vescovi AL, et al. 2007. Systematic studies of a selfassembling peptide nanofiber scaffold with other scaffolds. $J$ Nanosci Nanotechnol, 7:424-34.

Genove E, Shen C, Zhang S, et al. 2005. The effect of functionalized selfassembling peptide scaffolds on human aortic endothelial cell function. Biomaterials, 26:3341-51.

Ghosh S, Spagnoli GC, Martin I, et al. 2005. Three-dimensional culture of melanoma cells profoundly affects gene expression profile: a high density oligonucleotide array study. J Cell Physiol, 204:522-31.

Gomez-Lechon MJ, Jover R, Donato T, et al. 1998. Long-term expression of differentiated functions in hepatocytes cultured in three-dimensional collagen matrix. J Cell Physiol, 177:553-62.

Guo J, Su H, Zeng Y, et al. 2007. Reknitting the injured spinal cord by selfassembling peptide nanofiber scaffold. Nanomedicine, 3:311-21.

Haddish-Berhane N, Rickus JL, Haghighi K. 2007. The role of multiscale computational approaches for rational design of conventional and nanoparticle oral drug delivery systems. Int J Nanomedicine, 2:315-31.

Holmes T C, de Lacalle S, Su X, et al. 2000. Extensive neurite outgrowth and active synapse formation on self-assembling peptide scaffolds Proc Natl Acad Sci U S A, 97:6728-33.

Horii A, Wang X, Gelain F, et al. 2007. Biological designer self-assembling peptide nanofiber scaffolds significantly enhance osteoblast proliferation, differentiation and 3-D migration. PLOS ONE, 2:e190.

Jansen EJ, Sladek RE, Bahar H, et al. 2005. Hydrophobicity as a design criterion for polymer scaffolds in bone tissue engineering. Biomaterials, 26:4423-31

Kisiday J, Jin M, Kurz B, et al. 2002. Self-assembling peptide hydrogel fosters chondrocyte extracellular matrix production and cell division: implications for cartilage tissue repair. Proc Natl Acad Sci USA, 99:9996-10001.

Kreis T, Vale R. 1999. Guide book to the extracellular matrix, anchor, and adhesion proteins. Oxford: Oxford University Press.

Mann-Metzer P, Yarom Y. 2002. Pre- and postsynaptic inhibition mediated by $\mathrm{GABA}(\mathrm{B})$ receptors in cerebellar inhibitory interneurons. J Neurophysiol, 87:183-90.

Manwaring ME, Walsh JF, Tresco PA. 2004. Contact guidance induced organization of extracellular matrix. Biomaterials, 25:3631-8. 
Nagai Y, Unsworth L D, Koutsopoulos S, et al. 2006. Slow release of molecules in self-assembling peptide nanofiber scaffold. J Control Release, 115:18-25.

Panseri S, Cunha C, Lowery J, et al. 2008. Electrospun micro- and nanofiber tubes for functional nervous regeneration in sciatic nerve transections. BMC Biotechnol, 8:39.

Pham QP, Sharma U, Mikos AG. 2006. Electrospinning of polymeric nanofibers for tissue engineering applications: a review. Tissue Eng, 12:1197-211.

Powell HM, Boyce ST. 2008. Fiber density of electrospun gelatin scaffolds regulates morphogenesis of dermal-epidermal skin substitutes. J Biomed Mater Res A, 84:1078-86.

Riboldi SA, Sadr N, Pigini L, et al. 2008. Skeletal myogenesis on highly orientated microfibrous polyesterurethane scaffolds. J Biomed Mater Res A, 84:1094-101.

Ricard-Blum S, Dublet B, Van Der Rest M. 2000. Unconventional Collagens: Types VI, VII, VIII, IX, X, XIV, XVI and XIX. Oxford: Oxford University Press.

Rubenstein D, Han D, Goldgraben S, et al. 2007. Bioassay chamber for angiogenesis with perfused explanted arteries and electrospun scaffolding. Microcirculation, 14:723-37.

Sieminski AL, Was AS, Kim G, et al. 2007. The stiffness of threedimensional ionic self-assembling peptide gels affects the extent of capillary-like network formation. Cell Biochem Biophys, 49:73-83.

Silva GA, Czeisler C, Niece KL, et al. 2004. Selective differentiation of neural progenitor cells by high-epitope density nanofibers. Science, 303:1352-5

Srouji S, Kizhner T, Suss-Tobi E, et al. 2008. 3-D Nanofibrous electrospun multilayered construct is an alternative ECM mimicking scaffold. J Mater Sci Mater Med, 19:1249-55.
Sun T, Mai S, Norton D, et al. 2005. Self-organization of skin cells in three-dimensional electrospun polystyrene scaffolds. Tissue Eng, 11:1023-33.

Tysseling-Mattiace VM, Sahni V, Niece KL, et al. 2008. Self-assembling nanofibers inhibit glial scar formation and promote axon elongation after spinal cord injury. J Neurosci, 28:3814-23.

Venugopal J, Low S, Choon AT, et al. 2008. Electrospun-modified nanofibrous scaffolds for the mineralization of osteoblast cells. J Biomed Mater Res A, 85:408-17.

Walboomers XF, Jansen JA. 2001. Cell and tissue behavior on microgrooved surfaces. Odontology, 89:2-11.

Wan Y, Wang Y, Liu Z, et al. 2005. Adhesion and proliferation of OCT-1 osteoblast-like cells on micro- and nano-scale topography structured poly(L-lactide). Biomaterials, 26:4453-9.

$\mathrm{Xu} \mathrm{C}$, Yang F, Wang S, et al. 2004. In vitro study of human vascular endothelial cell function on materials with various surface roughness. J Biomed Mater Res A, 71:154-61.

Xu W, He X, Sarvestani AS, et al. 2007. Effect of a low-molecular-weight cross-linkable macromer on electrospinning of poly(lactide-co-glycolide) fibers. J Biomater Sci Polym Ed, 18:1369-85.

Yoshimoto H, Shin YM, Terai H, et al. 2003. A biodegradable nanofiber scaffold by electrospinning and its potential for bone tissue engineering. Biomaterials, 24:2077-82.

Zhang S. 2003. Fabrication of novel biomaterials through molecular selfassembly. Nat Biotechnol, 21:1171-8.

Zhang Y, Lim CT, Ramakrishna S, et al. 2005. Recent development of polymer nanofibers for biomedical and biotechnological applications. J Mater Sci Mater Med, 16:933-46.

Zong X, Bien H, Chung C Y, et al. 2005. Electrospun fine-textured scaffolds for heart tissue constructs. Biomaterials, 26:5330-8. 